\title{
Using animal models to understand cancer pain in humans
}

Gillian L. Currie, Emily S. Sena, Marie T. Fallon, Malcolm R. Macleod* and Lesley A. Colvin

Gillian L. Currie, PhD

Department of Clinical Neurosciences, Chancellors Building University of Edinburgh, Edinburgh, UK

Phone: +44-131-242-6348

Email: gillian.currie@ed.ac.uk

Emily S. Sena, $\mathrm{PhD}$

Department of Clinical Neurosciences, Chancellors Building

University of Edinburgh, Edinburgh, UK

Phone: +44-131-242-6348

emily.sena@ed.ac.uk

Marie T. Fallon, MB ChB, MD, FRCP

Edinburgh Cancer Research UK Centre

University of Edinburgh, Western General Hospital, Edinburgh, UK

Phone: +44-131-777-3518

Email: marie.fallon@ed.ac.uk

\section{* Corresponding Author:}

Malcolm R. Macleod, MBChB, PhD, FRCP

Department of Clinical Neurosciences, Chancellors Building

University of Edinburgh, Edinburgh, UK

Phone: +44-131-242-6348

Email:malcolm.macleod@ed.ac.uk

Lesley A. Colvin, MB ChB, BSc (Med Sci), PhD, FRCA, FFPMRCA, FRCPE

Department of Anaesthesia, Critical Care and Pain Medicine

Western General Hospital, Edinburgh, UK

Phone: +44-131-537-1646

Email: lesley.colvin@ed.ac.uk

(Word count: 3897$)$

Keywords: Cancer pain, Animal models, Neuropathic pain, Inflammation, Opioid treatment

\section{Abstract}


Cancer pain is not a single entity but a complex pain state involving different pain syndromes, with inflammatory, neuropathic, compressive, and ischaemic mechanisms. Current therapeutic regimens are based largely on opioids, although opioid treatment can lead to many side effects. Studies using animal models of cancer pain are aimed at understanding cancer pain and developing novel therapies. The most frequently reported models are of bone cancer pain, predominantly modelling pain associated with tumour growth within bone marrow. Here we summarise recent findings from studies using animal models of cancer pain and discuss the methodological quality of these studies.

\section{Introduction}

Due to current therapies, cancer patients have increased survival such that both cancer pain and cancer treatment-associated pain are growing clinical problems. Cancer pain is a complex and varied pain state, potentially involving different pain syndromes, with inflammatory, neuropathic, compressive, and ischaemic mechanisms. The current therapeutic regimen for cancer pain is based on the World Health Organisation (WHO) analgesic ladder, where the mainstay of treatment is opioids. Opioid-related side effects, however, can limit effective treatment.

Cancer pain is modelled in animals to better understand fundamental mechanisms and to improve treatment strategies. The International Association of Pain defines pain as "an unpleasant sensory and emotional experience associated with actual or potential tissue damage, or described in terms of such damage." The subjective nature of pain renders the modelling of pain and the interpretation of pain-related behaviours challenging in animals.

We performed a search of the literature using animal models of cancer pain published between July 2012 and August 2013. Models of bone cancer, melanoma, head and neck cancer, and peritoneal carcinomatosis were reported. This review summarises recent findings in an evaluation of how animal models are facilitating our understanding of cancer pain in humans and assesses the methodological quality of these studies.

\section{Bone cancer pain}


Pain related to tumour in bones is most commonly a consequence of metastatic disease, but can also arise from primary bone tumours such as sarcoma. In the clinic, pain from bone metastases is among the most commonly reported type of cancer pain [1]. The most frequently reported cancer pain models are of bone pain, predominantly modelling pain associated with tumour growth within bone marrow. Earlier models used the systemic administration of cancer cells, only some of which would lodge in the bone marrow. More recently, models have been developed which involve direct implantation of cancer cells within the bone marrow. These models allow assessment of site-specific pain-related behaviours and avoid the confounding effects of cancer metastases to other organs.

There are many different models of bone cancer pain which use different cell lines and different sites of tumour cell implantation [2]. A new model was recently characterised where rat breast cancer cells were implanted into the shaft of femur at the third trochanter level [3]. Pain-related behaviours were observed over 21 days, measured as a decrease in limb use score, increased spontaneous flinching and guarding time, and decreased paw withdrawal threshold. These pain-related behaviours may reflect the clinical phenomena of ongoing pain, movement-evoked pain, and breakthrough pain that may occur spontaneously or are related to movement. Spontaneous pain is particularly difficult to treat because of rapid onset [4].

Because pain is an experience, animal models rely on observing pain-related behaviours. These often involve reflex responses to a given stimulus, such as mechanical (e.g., paw withdrawal threshold to von Frey filaments) or thermal (e.g., Hargreaves test) stimuli. These evoked reflex responses are often criticised because they do not reflect the major clinical problems of spontaneous and ongoing pain. There has been a shift recently towards observations of spontaneous activities such as limb use, flinching, and guard time. Future studies need to identify whether the observational assessments are more suitable measures of pain-related behaviour and whether results using these tests are more likely to translate to the clinic. Further, it would be useful to establish whether more complex operant behavioural assays that use a reward-escape paradigm, such as the conditioned place preference assay, might be used to measure ongoing pain [5]. However, no study in our recent meta-analysis of animal models of bone cancer pain used such outcomes [2]. In the clinic, a more direct assessment of the experience of pain is possible, with patients 
describing pain intensity using the Numerical Rating Scale, the Visual Analog Scale, the Iowa Pain Thermometer Scale, and the Faces Pain Scale [6]. Unfortunately, the reporting of a level of pain does not address whether a treatment is effective against individual components of pain (evoked, ongoing, or spontaneous) [7]. More specific assessment of pain phenotypes in patients is likely to facilitate the translational process.

Previous animal models were of relatively short duration compared to the clinical situation, as they relied on the injection of a large number of cancer cells, often resulting in rapid deterioration of animal health and extensive bone destruction, necessitating early euthanasia of animals. A recent study refined a rat model of bone cancer pain to allow longer-term assessment [8]. A range of concentrations of AT3B prostate cancer cells were injected into the tibia. Following the injection of 40,000 cells, animals maintained relatively good health for up to 90 days, allowing more extensive analysis of pain-related behaviours and bone destruction. Animals displayed bilateral mechanical allodynia and thermal hyperalgesia that was fully developed between days 14 and 21 post tumour cell implantation. Histological and radiological assessments showed that tumour growth was confined to the inoculated tibia. Interestingly, the hindpaw sensitivity of this animal model appeared to spontaneously remit at day 28 and remained absent until the end of the experiment at 90 days. However, this reversal of pain-related behaviours was abolished by administration of the opioid receptor antagonist naloxone. It is possible that up-regulation of endogenous opioid signalling pathways underlie the spontaneous reversal of hindpaw sensitivity in later stages of bone cancer. Whether this occurs in the clinical situation requires further investigation.

Bilateral pain-related behaviours, as described by Muralidharan et al., have been observed in other bone cancer pain models and are also known as 'mirror-image pain' [9--11]. This pain-related behaviour is thought to model aspects of pain described by cancer patients, where patients experience increased sensitivity in areas not directly overlying a tumour site. The description of this pain phenomenon in animal models confirms shared characteristics with the clinical pain state. It is possible that bilateral allodynia is the result of bilateral changes that occur in the spinal cord, such as the activation of glial cells and altered expression of cytokines and receptors. 


\section{Glial cells in the spinal cord in bone cancer pain}

Astrocyte activation in bone cancer was first described by Schwei et al. [12], and activation of spinal astrocytes and microglia has since been reported in numerous animal models. Glial cells may contribute to bone cancer pain by releasing pro-inflammatory cytokines such as interleukin-1 $\beta$ (IL-1 $\beta$ ) and tumour necrosis factor- $\alpha$ (TNF- $\alpha$ ) [13--15]. Further, extracellular signal-regulated kinase (ERK) activation in spinal glial cells may be involved in the initiation and maintenance of bone cancer pain [16]. Phosphorylation of ERK1/2 was increased in microglia and astrocytes in bone cancer pain, and administration of ERK inhibitors PD98059 and UO126 inhibited mechanical allodynia, identifying ERK as a potential therapeutic target.

The microglia CX3C chemokine receptor 1 (CX3CR1)/p38 mitogen-activated protein (MAP) kinase signalling pathway may also play an important role in bone cancer pain. CX3CR1 and p38 MAP kinase were increased in the spinal cord in a rat model of bone cancer pain [17], and a neutralising antibody to CX3CR1 delayed the onset of mechanical allodynia and attenuated established mechanical allodynia, as well as inhibiting microglia activation and p38 MAP kinase.

Proinflammatory cytokines released by astrocytes may be therapeutic targets for bone cancer pain. Administration of lipoxin A4, lipoxin B4, or aspirin-triggered-15-epi-lipoxin A4 (ATL) alleviated mechanical allodynia in bone cancer pain animals [18]. Intrathecal administration of ATL gave 6 hours of analgesia and also attenuated pain when administered intravenously. In the spinal cord the lipoxin receptor (ALX) was mainly colocalised with astrocytes. ATL administration reduced the expression of the cytokines IL$1 \beta$ and TNF- $\alpha$ in the spinal cord.

Mechanical allodynia in a rat model of bone cancer pain was associated with increased Damino acid oxidase (DAAO) expression and DAAO enzymatic activity [19]. Activation of astrocytes in the spinal cord was also observed. A DAAO inhibitor CBIO (5-chlorobenzo[d]isoxazol-3-ol) attenuated mechanical allodynia and reduced both the production of spinal hydrogen peroxide and astrocyte activation. Similarly, administration of a DAAO gene silencer, small interfering RNA (siRNA)/DAAO, attenuated mechanical allodynia for at least 6 days. 
It is clear that glial cells do play a role in bone cancer pain and that this is a complex relationship involving many pathways. It is not known whether targeting a single pathway will be sufficient to relieve a specific cancer pain in the clinic.

\section{Dorsal root ganglia in bone cancer pain}

In addition to changes in the spinal cord, animal studies have identified changes in the dorsal root ganglia (DRG) that may present new analgesic targets. The P2X3 receptor was up-regulated in DRG neurons in a rat model of bone cancer pain [20], and daily intrathecal administration of the P2X3 antagonist A-317491 attenuated spontaneous ambulatory pain and mechanical hyperalgesia. Furthermore, the study reported increased functional activity of $\mathrm{P} 2 \mathrm{X} 3$ receptors in dissociated DRG neurons. This study identifies the $\mathrm{P} 2 \mathrm{X} 3$ receptor as a potential target in bone cancer pain. Another study investigated the analgesic efficacy of A-317491 in a mouse model of bone cancer pain [21]. Chronic administration of A317491 transiently attenuated pain-related behaviours 10 days after tumour cell implantation but did not attenuate pain-related behaviours in advanced bone cancer pain.

Another study investigated the therapeutic potential of potassium voltage-gated channel subfamily KQT (KCNQ channels) in DRG neurons. A decrease in expression of KCNQ2 and KCNQ3 channel proteins was observed in bone cancer pain rats, as well as decreased M-current, which may increase excitability in DRG neurons and lead to hyperalgesia [22]. Activation of $\mathrm{KCNQ} / \mathrm{M}$ channels with retigabine inhibited the hyperexcitability of the DRG neurons and attenuated mechanical allodynia and thermal hyperalgesia. The targeting of KCNQ2 channels may be a promising step in the treatment of bone cancer pain in the clinic.

\section{Tumour cells in bone cancer pain}

The type and severity of cancer pain may be dependent upon the type of tumour. Identifying and targeting factors released by tumour cells and associated immune cells involved in the generation of pain may offer tumour-specific analgesia. Inflammatory mediators released by tumour cells include endothelin-1 (ET-1), nerve growth factor (NGF), and prostaglandin E2 ( $\left.\mathrm{PGE}_{2}\right)$ [23]. Tumour-associated macrophages, mast cells, 
neutrophils, and T lymphocytes also release inflammatory mediators, including IL-1 $\beta$, TNF- $\alpha$, and NGF [23]. NCTC 2472 tumour cells release the cytokine chemokine (C-C motif) ligand 2 (CCL2) in culture and in a mouse model of bone cancer pain [24]. Administration of the selective C-C chemokine receptor type 2 (CCR2) antagonist RS 504393 inhibited thermal and mechanical hyperalgesia, but not mechanical allodynia, in a NCTC 2472 model of bone cancer pain. Thermal hyperalgesia was also inhibited by a CCL2 neutralising antibody. In contrast, B16-F10 cells did not alter CCL2 levels in a mouse model, and RS 504393 did not attenuate pain-related behaviours. This study suggests that it may be possible to identify tumour types likely to respond to CCR2 antagonists and that tailored targeted approaches to therapy may be beneficial.

In a rat model where MRMT-1 rat mammary gland carcinoma cells were injected into the tibia [25], tumour cells expressed the A3 adenosine receptor (A3AR), and administration of the A3AR agonist, 2-chloro- $N^{6}$-(3-iodobenzyl)-adenosine-5te $N$-methyl-uronamide (CIIB-MECA), reduced tumour growth and mechanical allodynia. A summary of molecular changes reported in recent literature in animal models of bone cancer are shown in Table 1.

\section{Table 1 here}

\section{Bone destruction in bone cancer pain}

Tumours growing within the bone can lead to significant bone remodelling, and associated skeletal-related events represent a major clinical problem. An imagining study using magnetic resonance imaging (MRI) and micro X-ray computed tomography $(\mu \mathrm{CT})$ aimed to determine whether pain-related behaviours are directly correlated to tumour progression and bone remodelling in a rat model of bone cancer pain [26]. Results demonstrated that increased tumour volume correlated with increased mechanical allodynia and weightbearing deficits. Bone degradation for trabecular and cortical bone showed different profiles, where trabecular degradation occurred earlier and cortical later. In both trabecular and cortical bone, increased bone degradation strongly correlated with increased 
mechanical allodynia and weight-bearing deficits, albeit less aggressively in cortical bone. Trabecular bone volume was affected at an early stage of tumour development, whereas cortical bone degradation occurred more in proportion to the tumour growth. Clinically, these imaging techniques may be useful in detecting tumour progression before pain occurs. In particular, detecting trabecular bone degradation at an early stage may enable pre-emptive treatment for at-risk patients.

Another recent study using a mouse model of bone cancer pain showed that greater bone degradation correlated with increased pain-related behaviours in both sexes [27]. Although there was no difference in bone degradation between male and female mice, tumour size, measured using bioluminescence, was larger in female animals compared to males at day 14 post mammary cancer cell implantation into the femur.

\section{Cannabinoids in bone cancer pain}

Cannabinoids have shown analgesic efficacy in various chronic pain conditions [28] and could also be useful in the treatment of cancer pain. The general cannabinoid agonist WIN 55,212-2 was previously shown to attenuate pain-related behaviours in a rat model of bone cancer pain. This analgesia was reversed by both CB1 and CB2 antagonists [29]. The CB2 agonist JWH015 also attenuated bone cancer pain in a mouse model [30]. The analgesic potential in bone cancer pain of a CB2 agonist, MT178, was recently assessed [31]. MT178 attenuated mechanical allodynia and, importantly, did not induce locomotor disturbance and catalepsy, which was observed following administration of the nonselective CB agonist WIN 55,212-2. In another study involving the implantation of spontaneously occurring murine mammary cell line (66.1) into the femur of mice, administration of the CB2 agonist JWH015 for 7 days attenuated pain-related behaviours, bone loss, and cancer proliferation [32]. These promising results highlight the potential value of cannabinoids in bone cancer pain.

\section{Opioids in bone cancer pain}

Opioids are one of the primary treatments used in bone cancer patients to control background pain, but show less efficacy in controlling breakthrough pain due to the fast 
onset of this component. [33]. Many opioids, including morphine and oxycodone, act on $\mu$-opioid receptors, which are expressed in many brain regions involved in pain modulation. A previous study showed that morphine was less effective than oxycodone at attenuating pain-related behaviours in a mouse model of bone cancer [34]. To determine the reasons for this, functional changes in the $\mu$-opioid receptor were investigated in a mouse model of bone cancer pain [35]. Results suggest that the total number of $\mu$-opioid receptors on tissue membranes was reduced in several pain-related regions: the periaqueductal grey matter (PAG), region ventral to the PAG (vPAG), mediodorsal thalamus (mTH), ventral thalamus, and spinal cord. Furthermore, the functional activation of $\mu$-opioid receptors by oxycodone and morphine was attenuated in several regions related to pain signalling. There was less attenuation of the $\mu$-opioid receptor activation by oxycodone compared to morphine. Consistent with these results, oxycodone demonstrated a greater analgesic potency than morphine following intracerebroventricular administration. Modulation of $\mu$-opioid receptor function may be one of the mechanisms underlying the distinct analgesic effects of oxycodone and morphine in bone cancer pain.

A study using a rat model of bone cancer pain utilised the paw withdrawal threshold to noxious heat to assess the analgesic efficacy of morphine and flupirtine given alone and in combination [36]. Flupirtine is a KCNQ channel activator which increases M-current in neurons and therefore decreases neuronal activity. Both morphine and flupirtine reversed bone cancer-induced thermal hyperalgesia. Results showed that flupirtine combined with morphine treatment improved the ability of morphine to reverse hyperalgesia. This study suggests that this combination therapy could be useful in the clinic. However, it is worth considering that the study assessed analgesic efficacy only in thermal hyperalgesia, and more research is necessary to look at the effect on other pain-related behaviours before this is taken forward to the clinic.

The use of opioids in cancer pain is limited by side effects, including the induction of tolerance to analgesia. This has been demonstrated in animal models, where a series of bidaily morphine injections result in potent analgesia but tolerance quickly develops. Damino acid oxidase (DAAO) is expressed in the spinal cord, predominantly in astrocytes. A recent study assessed whether DAAO contributes to bone cancer pain and morphine analgesic tolerance [19]. Spinal DAAO expression was up-regulated in a rat model of 
bone cancer pain which correlated with development of mechanical allodynia. Administration of CBIO attenuated mechanical allodynia. In addition, bi-daily injections of $\mathrm{CBIO}$ over 7 days did not result in tolerance to $\mathrm{CBIO}$ analgesia and prevented morphine tolerance to analgesia. Furthermore, CBOI administration with morphine resulted in additive analgesic effect. As such, this study identifies a potentially useful adjuvant to morphine.

\section{Melanoma model of cancer pain}

A study using a mouse model of melanoma, where B16 mouse melanoma cells were injected subcutaneously into the plantar pad, demonstrated that these mice developed cold, mechanical, and heat hyperalgesia in the tumour-bearing paw [37]. In the same study, CCL-11 sarcoma cells were injected into and around the calcaneus bone. Both cancer pain models displayed 'mirror image pain' with hyperalgesia in the contralateral paw. The authors set out to assess whether the GTP cyclohydrolase (GCH1) plays a role in cancer pain. The GCH1 inhibitor diaminohydroxypyrimidine (DAHP) did not attenuate cold, mechanical, or thermal hyperalgesia in either the melanoma or bone cancer pain models. Inhibition of GCH1 reduced thermal hyperalgesia of the contralateral paw in both models. Combined administration of DAHP and morphine extended the analgesic effects of morphine without increasing morphine-induced respiratory depression. The authors suggest that DAHP may be a useful adjuvant in cancer pain.

The analgesic efficacy of an inhibitor of the NGF receptor Tropomyosin receptor kinase (Trk) A, IPTRK3, was investigated in a mouse model of melanoma [38]. Chronic administration of IPTRK3 inhibited mechanical allodynia and the number of spontaneous flinches but did not attenuate thermal hyperalgesia. Administration of IPTRK3 was also associated with a reduction in increased paw volume, which may represent a reduction of tumour growth.

\section{Models of head and neck cancer}

Risk factors for head and neck cancer include tobacco and alcohol use, human papillomavirus (HPV) infection, and Epstein-Barr virus infection. These different 
pathologies may result in different sites of head and neck cancer. For example, HPVassociated cancers often occur in the oropharynx [39]. Head and neck cancer patients may suffer from pain throughout the disease course. In addition to pain, patients suffer from debilitation of oral function which worsens as the disease progresses. Although first animal models of head and neck cancer relied on reflex responses such as paw withdrawal to measure pain, three novel mouse models have been used to model the temporal progression and functional impairment experienced by patients [40]. In the acute pain model, mice were injected with supernatant from human oral squamous cell carcinoma (SCC) into the tongue. Cancer-induced functional impairment or mechanical allodynia was measured by increased gnaw time, which began at 45 minutes post-SCC supernatant injection and lasted around 24 hours. Trypsin injection also resulted in allodynia in mice. The authors suggest that trypsin released from cancer cells may be responsible for inducing acute cancer pain. Mechanical allodynia induced by SCC supernatant or trypsin was absent in PAR2-/- mice, supporting the hypothesis that acute cancer pain is PAR2dependent. A persistent oral cancer pain model was generated by injection of human oral SCC cells into the tongue. These mice developed functional allodynia at day 18 posttumour cell implantation which persisted for 8 days. This allodynia was attenuated by soybean trypsin inhibitor (SBTI), indicating that trypsin may also play a role in persistent cancer pain. A model of chronic cancer pain was established by including the carcinogen 4-Nitroquinoline 1-oxide (NoK) in the drinking water of mice. This resulted in functional allodynia that began 4--6 weeks post-4NQO treatment and worsened over the remaining 2 months. Functional allodynia did not develop in PAR2-/- mice despite cancer progression. In the persistent and chronic pain models, PAR2-immunoreactivity and mRNA expression were significantly up-regulated in the trigeminal ganglia but not in the acute pain model. This suggests that PAR2 plays a role in head and neck cancer pain, and it provides evidence that functional behavioural tests are useful in animal pain studies. Analgesics which show efficacy against functional outcomes, such as gnaw time, may be more likely to translate to efficacy in the clinic.

\section{A model of peritoneal carcinomatosis}


Abdominal pain resulting from peritoneal carcinomatosis is often difficult to treat, as it does not always respond to opioids. To understand peritoneal carcinomatosis cancer pain, a new model was developed in which peritoneal-seeding gastric cancer cells (60As6) were implanted into the abdominal cavity [41]. These mice showed hypersensitivity of the abdomen to mechanical stimulation and spontaneous visceral pain-related behaviour (as measured by hunching). Increased C-Fos-positive cells in the spinal cord, increased substance P-positive neurons in the DRG, and decreased $\mu$-opioid receptor expression, mainly in substance P-positive neurons, was observed in the mice. Similar to clinical observations, a higher dose of morphine was also required to attenuate hyperalgesia in this model compared to models of acute pancreatitis or inflammatory pain. These results suggest that decreased $\mu$-opioid receptor expression in substance P-positive neurons in the DRG may correspond to a reduction in morphine-induced analgesia in peritoneal carcinomatosis. This model appears to reflect the clinical condition of peritoneal carcinomatosis, and it may be useful in assessing analgesic interventions in future studies.

\section{Methodological quality of studies}

Our previous systematic review of studies using animal models of bone cancer pain suggested that methodological quality has a significant impact on reported behavioural outcomes. Blinding and randomisation were associated with smaller observed differences in behavioural outcomes between tumour-bearing and control animals [2]. This is consistent with findings in other fields, where studies that do not report such measures report inflated treatment effects [42--45]. It is important to consider the validity of the recent studies included in this review (studies published from July 2012 to August 2013) if they are to be used to understand cancer pain in humans. Assessment of the reporting of measures to reduce the risk of bias of studies included in this review indicates that $58 \%$ of studies report blinded assessment of outcome and $22 \%$ report random allocation to group, although no study reported allocation concealment or a sample size calculation. These results are an improvement compared to our systematic review (up to and including July 2011), where only $31 \%$ of studies were blinded and $11 \%$ were randomised. However, consistently, no study reported a sample size calculation [2]. The lack of reporting of sample size calculations in this field is troubling. Performing sample size calculations will 
reduce the number of animals wasted in both underpowered and unnecessarily large experiments. Further improvement in methodological quality is necessary. Reducing potential sources of bias will increase the likelihood that the results from these studies reliably inform the translational process and clinical research.

\section{Conclusion}

Animal models of cancer pain appear to reflect the complex pain state observed in the clinic with mechanical, thermal, and spontaneous pain-related behaviours and bilateral allodynia. Animal studies have identified potential therapeutic targets. Results indicate that it is unlikely that a single treatment will target all of the different pain-related behaviours and that combined treatment strategies should be investigated in animal models. Finally, methodological quality of studies must improve if results are to inform reliably the treatment of human cancer pain.

\section{Compliance with Ethics Guidelines}

\section{Conflict of Interest}

Dr. Gillian L. Currie, Dr. Emily S. Sena, Dr. Marie T. Fallon, Dr. Malcolm R. Macleod and Dr. Lesley A. Colvin each declare no potential conflicts of interest.

\section{Human and Animal Rights and Informed Consent}

This article does not contain any studies with human or animal subjects performed by any of the authors.

\section{References}

- Of importance

1. Grond S, Zech D, Diefenbach C, Radbruch L, Lehmann KA. Assessment of cancer pain: a prospective evaluation in 2266 cancer patients referred to a pain service. Pain. 1996;64(1):107-14.

2. Currie GL, Delaney A, Bennett MI, Dickenson AH, Egan KJ, Vesterinen HM et al. Animal models of bone cancer pain: systematic review and meta-analyses. Pain. 2013;154(6):917-26. doi:10.1016/j.pain.2013.02.033.

- This systematic review and meta-analysis describing in vivo modelling of bone cancer pain showed for the first time that methodological quality has a significant impact on reported behavioural outcomes in in vivo studies of pain. Blinding and randomisation were associated with smaller observed differences in behavioural outcomes between tumour-bearing and control animals. 
3. Gui Q, Xu C, Zhuang L, Xia S, Chen Y, Peng P et al. A new rat model of bone cancer pain produced by rat breast cancer cells implantation of the shaft of femur at the third trochanter level. Cancer Biol Ther. 2013;14(2):193-9. doi:10.4161/cbt.23291.

4. Laird BJ, Walley J, Murray GD, Clausen E, Colvin LA, Fallon MT. Characterization of cancer-induced bone pain: an exploratory study. Support Care Cancer. 2011;19(9):1393-401. doi:10.1007/s00520-010-0961-3.

5. Vierck CJ, Hansson PT, Yezierski RP. Clinical and pre-clinical pain assessment: are we measuring the same thing? Pain. 2008;135(1-2):7-10. doi:10.1016/j.pain.2007.12.008.

6. Buga S, Sarria JE. The management of pain in metastatic bone disease. Cancer Control. 2012;19(2):154-66.

7. Sikandar S, Dickenson AH. II. No need for translation when the same language is spoken. Br J Anaesth. 2013;111(1):3-6. doi:10.1093/bja/aet210.

- This editorial explored the reasons for the apparent failure of results from studies using animal models of chronic pain to translate to effective therapies to the clinic.

8. Muralidharan A, Wyse BD, Smith MT. Optimization and characterization of a rat model of prostate cancer-induced bone pain using behavioral, pharmacological, radiological, histological and immunohistochemical methods. Pharmacol Biochem Behav. 2013;106:33-46. doi:10.1016/j.pbb.2013.02.020.

9. Mao-Ying QL, Zhao J, Dong ZQ, Wang J, Yu J, Yan MF et al. A rat model of bone cancer pain induced by intra-tibia inoculation of Walker 256 mammary gland carcinoma cells. Biochem Biophys Res Commun. 2006;345(4):1292-8. doi:10.1016/j.bbrc.2006.04.186.

10. Lee BH, Seong J, Kim UJ, Won R, Kim J. Behavioral characteristics of a mouse model of cancer pain. Yonsei Med J. 2005;46(2):252-9.

11. Miao XR, Gao XF, Wu JX, Lu ZJ, Huang ZX, Li XQ et al. Bilateral downregulation of Nav1.8 in dorsal root ganglia of rats with bone cancer pain induced by inoculation with Walker 256 breast tumor cells. BMC Cancer. 2010;10:216. doi:10.1186/14712407-10-216.

12. Schwei MJ, Honore P, Rogers SD, Salak-Johnson JL, Finke MP, Ramnaraine ML et al. Neurochemical and cellular reorganization of the spinal cord in a murine model of bone cancer pain. J Neurosci. 1999;19(24):10886-97.

13. Zhang RX, Liu B, Wang L, Ren K, Qiao JT, Berman BM et al. Spinal glial activation in a new rat model of bone cancer pain produced by prostate cancer cell inoculation of the tibia. Pain. 2005;118(1-2):125-36. doi:10.1016/j.pain.2005.08.001.

14. Hald A, Nedergaard S, Hansen RR, Ding M, Heegaard AM. Differential activation of spinal cord glial cells in murine models of neuropathic and cancer pain. Eur J Pain. 2009;13(2):138-45. doi:10.1016/j.ejpain.2008.03.014.

15. Liu S, Yang J, Wang L, Jiang M, Qiu Q, Ma Z et al. Tibia tumor-induced cancer pain involves spinal p38 mitogen-activated protein kinase activation via TLR4-dependent mechanisms. Brain Res. 2010;1346:213-23. doi:10.1016/j.brainres.2010.05.014.

16. Wang XW, Li TT, Zhao J, Mao-Ying QL, Zhang H, Hu S et al. Extracellular signalregulated kinase activation in spinal astrocytes and microglia contributes to cancerinduced bone pain in rats. Neuroscience. 2012;217:172-81. doi:10.1016/j.neuroscience.2012.04.065. 
17. Hu JH, Yang JP, Liu L, Li CF, Wang LN, Ji FH et al. Involvement of CX3CR1 in bone cancer pain through the activation of microglia p38 MAPK pathway in the spinal cord. Brain Res. 2012;1465:1-9. doi:10.1016/j.brainres.2012.05.020.

18. Hu S, Mao-Ying QL, Wang J, Wang ZF, Mi WL, Wang XW et al. Lipoxins and aspirin-triggered lipoxin alleviate bone cancer pain in association with suppressing expression of spinal proinflammatory cytokines. J Neuroinflammation. 2012;9:278. doi:10.1186/1742-2094-9-278.

19. Huang JL, Chen XL, Guo C, Wang YX. Contributions of spinal D-amino acid oxidase to bone cancer pain. Amino Acids. 2012;43(5):1905-18. doi:10.1007/s00726-012-1390$\mathrm{z}$.

20. Wu JX, Xu MY, Miao XR, Lu ZJ, Yuan XM, Li XQ et al. Functional up-regulation of $\mathrm{P} 2 \mathrm{X} 3$ receptors in dorsal root ganglion in a rat model of bone cancer pain. Eur J Pain. 2012;16(10):1378-88. doi:10.1002/j.1532-2149.2012.00149.x.

21. Hansen RR, Nasser A, Falk S, Baldvinsson SB, Ohlsson PH, Bahl JM et al. Chronic administration of the selective $\mathrm{P} 2 \mathrm{X} 3, \mathrm{P} 2 \mathrm{X} 2 / 3$ receptor antagonist, A-317491, transiently attenuates cancer-induced bone pain in mice. Eur J Pharmacol. 2012;688(13):27-34. doi:10.1016/j.ejphar.2012.05.008.

22. Zheng Q, Fang D, Liu M, Cai J, Wan Y, Han JS et al. Suppression of KCNQ/M (Kv7) potassium channels in dorsal root ganglion neurons contributes to the development of bone cancer pain in a rat model. Pain. 2013;154(3):434-48. doi:10.1016/j.pain.2012.12.005.

23. Jimenez-Andrade JM, Mantyh WG, Bloom AP, Ferng AS, Geffre CP, Mantyh PW. Bone cancer pain. Ann N Y Acad Sci. 2010;1198:173-81. doi:10.1111/j.17496632.2009.05429.x.

24. Pevida M, Gonzalez-Rodriguez S, Lastra A, Hidalgo A, Menendez L, Baamonde A. CCL2 released at tumoral level contributes to the hyperalgesia evoked by intratibial inoculation of NCTC 2472 but not B16-F10 cells in mice. Naunyn Schmiedebergs Arch Pharmacol. 2012;385(11):1053-61. doi:10.1007/s00210-012-0787-2.

25. Varani K, Vincenzi F, Targa M, Paradiso B, Parrilli A, Fini M et al. The stimulation of $\mathrm{A}(3)$ adenosine receptors reduces bone-residing breast cancer in a rat preclinical model. Eur J Cancer. 2013;49(2):482-91. doi:10.1016/j.ejca.2012.06.005.

26. Dore-Savard L, Beaudet N, Tremblay L, Xiao Y, Lepage M, Sarret P. A microimaging study linking bone cancer pain with tumor growth and bone resorption in a rat model. Clin Exp Metastasis. 2013;30(2):225-36. doi:10.1007/s10585-012-9530-0.

27. Falk S, Uldall M, Appel C, Ding M, Heegaard AM. Influence of sex differences on the progression of cancer-induced bone pain. Anticancer Res. 2013;33(5):1963-9.

28. Lynch ME, Campbell F. Cannabinoids for treatment of chronic non-cancer pain; a systematic review of randomized trials. Br J Clin Pharmacol. 2011;72(5):735-44. doi:10.1111/j.1365-2125.2011.03970.x.

29. Cui JH, Kim WM, Lee HG, Kim YO, Kim CM, Yoon MH. Antinociceptive effect of intrathecal cannabinoid receptor agonist WIN 55,212-2 in a rat bone tumor pain model. Neurosci Lett. 2011;493(3):67-71. doi:10.1016/j.neulet.2010.12.052.

30. Gu X, Mei F, Liu Y, Zhang R, Zhang J, Ma Z. Intrathecal administration of the cannabinoid 2 receptor agonist JWH015 can attenuate cancer pain and decrease mRNA 
expression of the 2B subunit of N-methyl-D-aspartic acid. Anesth Analg. 2011;113(2):405-11. doi:10.1213/ANE.0b013e31821d1062.

31. Vincenzi F, Targa M, Corciulo C, Tabrizi MA, Merighi S, Gessi S et al. Antinociceptive effects of the selective CB2 agonist MT178 in inflammatory and chronic rodent pain models. Pain. 2013;154(6):864-73. doi:10.1016/j.pain.2013.02.007.

32. Lozano-Ondoua AN, Hanlon KE, Symons-Liguori AM, Largent-Milnes TM, Havelin JJ, Ferland HL, 3rd et al. Disease modification of breast cancer-induced bone remodeling by cannabinoid 2 receptor agonists. J Bone Miner Res. 2013;28(1):92-107. doi:10.1002/jbmr.1732.

33. Middlemiss T, Laird BJ, Fallon MT. Mechanisms of cancer-induced bone pain. Clin Oncol (R Coll Radiol). 2011;23(6):387-92. doi:10.1016/j.clon.2011.03.003.

34. Minami K, Hasegawa M, Ito H, Nakamura A, Tomii T, Matsumoto M et al. Morphine, oxycodone, and fentanyl exhibit different analgesic profiles in mouse pain models. $\mathrm{J}$ Pharmacol Sci. 2009;111(1):60-72.

35. Nakamura A, Hasegawa M, Minami K, Kanbara T, Tomii T, Nishiyori A et al. Differential activation of the mu-opioid receptor by oxycodone and morphine in painrelated brain regions in a bone cancer pain model. Br J Pharmacol. 2013;168(2):375-88. doi:10.1111/j.1476-5381.2012.02139.x.

36. Kolosov A, Goodchild CS, Williams ED, Cooke I. Flupirtine enhances the antihyperalgesic effects of morphine in a rat model of prostate bone metastasis. Pain Med. 2012;13(11):1444-56. doi:10.1111/j.1526-4637.2012.01502.x.

37. Pickert G, Myrczek T, Ruckert S, Weigert A, Haussler A, Ferreiros N et al. Inhibition of GTP cyclohydrolase reduces cancer pain in mice and enhances analgesic effects of morphine. J Mol Med (Berl). 2012;90(12):1473-86. doi:10.1007/s00109-012-0927-7.

38. Tabata M, Murata E, Ueda K, Kato-Kogoe N, Kuroda Y, Hirose M. Effects of TrkA inhibitory peptide on cancer-induced pain in a mouse melanoma model. J Anesth. 2012;26(4):545-51. doi:10.1007/s00540-012-1377-7.

39. Adelstein DJ, Ridge JA, Gillison ML, Chaturvedi AK, D'Souza G, Gravitt PE et al. Head and neck squamous cell cancer and the human papillomavirus: summary of a National Cancer Institute State of the Science Meeting, November 9-10, 2008, Washington, D.C. Head Neck. 2009;31(11):1393-422. doi:10.1002/hed.21269.

40. Lam DK, Dang D, Zhang J, Dolan JC, Schmidt BL. Novel animal models of acute and chronic cancer pain: a pivotal role for PAR2. J Neurosci. 2012;32(41):14178-83. doi:10.1523/jneurosci.2399-12.2012.

41. Suzuki M, Narita M, Hasegawa M, Furuta S, Kawamata T, Ashikawa M et al. Sensation of abdominal pain induced by peritoneal carcinomatosis is accompanied by changes in the expression of substance $\mathrm{P}$ and mu-opioid receptors in the spinal cord of mice. Anesthesiology. 2012;117(4):847-56. doi:10.1097/ALN.0b013e31826a4ac8.

42. Macleod MR, O'Collins T, Horky LL, Howells DW, Donnan GA. Systematic review and metaanalysis of the efficacy of FK506 in experimental stroke. J Cereb Blood Flow Metab. 2005;25(6):713-21. doi:10.1038/sj.jcbfm.9600064.

43. Macleod MR, van der Worp HB, Sena ES, Howells DW, Dirnagl U, Donnan GA. Evidence for the efficacy of NXY-059 in experimental focal cerebral ischaemia is 
confounded by study quality. Stroke. 2008;39(10):2824-9.

doi:10.1161/strokeaha.108.515957.

44. Rooke ED, Vesterinen HM, Sena ES, Egan KJ, Macleod MR. Dopamine agonists in animal models of Parkinson's disease: a systematic review and meta-analysis.

Parkinsonism Relat Disord. 2011;17(5):313-20. doi:10.1016/j.parkreldis.2011.02.010.

45. Vesterinen HM, Sena ES, ffrench-Constant C, Williams A, Chandran S, Macleod MR. Improving the translational hit of experimental treatments in multiple sclerosis. Mult Scler. 2010;16(9):1044-55. doi:10.1177/1352458510379612.

\section{Table Legend}

Table 1 Molecular changes in animal models of bone cancer. Abbreviations: cytokine chemokine (C-C motif) ligand 2 (CCL2); CX3C chemokine receptor 1 (CX3CR1); Damino acid oxidase (DAAO); extracellular signal-regulated kinase (ERK); interleukin-1 $\beta$ (IL-1 $\beta$ ); periaqueductal grey matter (PAG); region ventral to the mediodorsal thalamus $(\mathrm{mTH}) ;$ PAG (vPAG); tumour necrosis factor- $\alpha$ (TNF- $\alpha$ ) and ventral TH (vTH) 
Table 1 Molecular changes in animal models of bone cancer

\section{Molecular changes}

\begin{tabular}{|c|c|}
\hline PAG, vPAG, mTH and vTH & - Decreased $\mu$-opioid receptor expression \\
\hline Spinal cord & $\begin{array}{l}\text { - } \\
\text { - } \\
\text { - } \\
\text { - Increcreased phosphorion of spinal microglia and astrocytes } \\
\text { - Increased CX3CR1 expression } \\
\text { - Increased p38 MAP kinase expression } \\
\text { - Increased IL-1 } \beta \text { and TNF- } \alpha \text { expression } \\
\text { - Increased DAAO expression and DAAO enzymatic } \\
\text { activity }\end{array}$ \\
\hline Dorsal root ganglia & $\begin{array}{l}\text { - Increased P2X3 receptor expression } \\
\text { - Decreased KCNQ2 and KCNQ3 channels } \\
\text { expression }\end{array}$ \\
\hline Tumour cells & $\begin{array}{l}\text { - } \quad \text { NCTC } 2472 \text { tumour cells release CCL2 } \\
\text { - } \quad \text { MRMT-1 cells express A3 adenosine receptors }\end{array}$ \\
\hline
\end{tabular}

Table 1 Molecular changes in animal models of bone cancer

Abbreviations: cytokine chemokine (C-C motif) ligand 2 (CCL2); CX3C chemokine receptor 1 (CX3CR1); D-amino acid oxidase (DAAO); extracellular signal-regulated kinase (ERK); interleukin-1 $\beta$ (IL-1 $\beta$ ); periaqueductal grey matter (PAG); region ventral to the mediodorsal thalamus (mTH); PAG (vPAG); tumour necrosis factor- $\alpha$ (TNF- $\alpha$ ) and ventral TH (vTH) 